PROC. OF JSCE,

No. 285, MAY 1979

\title{
THE RIGIDITY OF JOINTS OF PRECAST ELEMENTS BY NON-BONDED PRESTRESSING
}

\author{
By Suresh Kumer REGMI* and Tada-aki TANABE**
}

\section{INTRODUCTION}

Due to the mechanisation of construction industry, precast elements are increasingly used today in every field of concrete construction. By the use of precast elements, works are accomplished in shorter duration than in cast-insitu construction.

A series of investigation of joints in precast concrete structures was initiated at the Research and Development Laboratories of the Portland Cement Association. Rostary ${ }^{1)}$ reported on the structural soundness of a continuity connection in double T-composite construction.

Kriz and Raths ${ }^{2}$ investigated the bearing strength of column heads supporting precast beams. This research demonstrated that the lateral reinforcement must be used to prevent longitudinal splitting of the column heads.

A second investigation Kriz and Raths ${ }^{3)}$ of the beam-to-column connections, reported by the same authors, concerned the development of the design criteria for the strength of the corbels which protrude from the face of the columns.

Kriz and Craston $^{4)}$ studied the strength and behaviour of bolted scarf joints in beams and columns. It was found that ultimate strength design principles of 1963 ACI code gives satisfactory results. Lafaugh and Magura ${ }^{5)}$ investigated the strength and stiffness of base plate connections between a precast column and its footing. Moment-rotation characteristics of the connections were determined.

Burns $\left.{ }^{6}\right)$ reported a test involving the use of rectangular precast prestressed concrete rods

* Graduate Student, Asian Institute of Technology.

** Associate Professor, Asian Institute of Technology as a means of providing continuity in composite constructions.

Schechter and Suarez ${ }^{7)}$ compared the conventional use of non-prestressed steel to the new technique of using prestressed steel in the connection of structures to the foundations. $\mathrm{He}$ has pointed that in the former type, steel changes from passive state to tension when the external forces acts on the structure. Consequently the amount of elongation depends on the magnitude of the tensil stress, the length of steel element, the bond characteristics of the same, etc.

One of the effective jointing method of precast pre-stressed concrete system, is considered to be the joint which utilizes member-prestressing force itself. In this type of joint, sometimes bond is given by grouting and sometimes it is not grouted. Even if it is grouted the bond between concrete and steel is rather weak and with higher stresses bonds tend to be broken easily and some portion of the joint is reasonably considered to be bondless.

On this point, no research work has been carried out in the past. In view of this, this research aims at:

(i) to develop the theory to predict the moment-rotation characteristics of nonbonded prestressed precast joints, i.e., to predict the rotation of the joint, separation of the joint and deflection of the non-bonded member for the given moment distribution,

(ii) to verify the accuracy of the proposed theory by experimentation.

\section{THEORETICAL CONSIDERATION}

To analyze a structure jointed by non-bonded prestressing, we must investigate the momentrotation relationship of the joint specifically. The following assumptions are made to develop the theoretical relationship. 


\section{(1) Assumptions}

1. The steel is assumed to be ideally nonbonded to the concrete. Hence, the strain and the force in the steel is same throughout the non-bonded length. As loading changes, strain in steel will change. This change of strain in steel is same throughout the nonbonded length.

2. Tension in concrete is neglected.

3. Strain distribution in concrete is assumed to be linear.

4. Shear deformation is neglected.

\section{(2) Governing Equations for the Solution of Non-bonded Pre-stressed Joint}

Fig. 1 shows the non-bonded length $L$ of the member jointed by pre-stressing. The co-ordinate axes $X, Y$ and $Z$ and positive directions of displacements are taken as shown in Fig. 1. Positive directions of the shear force $P$ and the moment $M$ acting at the edge of the non-bonded area are also shown in Fig. 1 . Let $R$ be the centre of rotation and $\theta$ be the angle through which the non-bonded member rotates at the joint. The anticlockwise rotation $\theta$ is taken as positive. For stress and strains, tension is taken as positive.

As the displacement along $Y$-axis is neglected,

$$
\begin{aligned}
& v=0 \\
& w=x \sin \theta-\left(x-x_{0}\right) \frac{\partial u}{\partial z}
\end{aligned}
$$

where $x_{0}$ is the distance of neutral surface from the $Z$ axis. Rotation of the joint $\theta$, being small

$$
\begin{aligned}
& w=x \theta-\left(x-x_{0}\right) \frac{\partial u}{\partial z} \\
& \epsilon_{z}^{\prime}=\frac{\partial w}{\partial z}
\end{aligned}
$$

From Eq. (2) and Eq. (3)

$$
\epsilon_{z}^{\prime}=-\frac{\partial}{\partial z}\left(\left(x-x_{0}\right) \frac{\partial u}{\partial z}\right)
$$

Rotation of the joint, $\theta$ in Eq. (2) is given by

$$
\theta=\frac{1}{d_{1}}\left[L \epsilon_{s}^{\prime}-\int_{0}^{L} \frac{\partial}{\partial z}\left\{\left(x_{0}-d_{1}\right) \frac{\partial u}{\partial z}\right\} d z\right]
$$

where $d_{1}$ is distance of pre-stressing steel measured from the centre of rotation, and $\epsilon_{s}^{\prime}$ and $\epsilon_{z}^{\prime}$ are increases of strain of steel and concrete due to external load respectively. Force equilibrium of the section considered gives

$$
B \int_{0}^{H} \sigma_{z} d x+E_{s} \epsilon_{s} A_{s}=0
$$

where $B$ and $H$ are the sectional dimensions along
$Y$ and $X$ axes respectively. The stress at any point $(x, y, z)$ in concrete along $Z$ direction is expressed as $\sigma_{z} . E_{s}, \epsilon_{s}$ and $A_{s}$ are the modulus of elasticity, strain and area of non-bonded steel.

Similarly moment equilibrium of the section around the gravity point of the steel gives,

$$
B \int_{0}^{H} \sigma_{z}\left(d_{1}-x\right) d x+M+P(L-z)=0
$$

Commonly used stress-strain relationship for concrete is parabolic, say

$$
\sigma_{z}=a \epsilon_{z}^{2}+b \epsilon_{z}+c
$$

which on substitution of Eq. (4) gives

$$
\begin{aligned}
\sigma_{z}= & a\left\{\frac{\partial}{\partial z}\left(\left(x_{0}-x\right) \frac{\partial u}{\partial z}\right)^{+} \epsilon_{z}{ }^{0}\right\}^{2} \\
& +b\left\{\frac{\partial}{\partial z}\left(\left(x_{0}-x\right) \frac{\partial u}{\partial z}\right)+\epsilon_{z}^{0}\right\}+c
\end{aligned}
$$

where $\epsilon_{z}{ }^{0}$ expresses initial concrete strain due to prestressing. Substitution of Eq. (8) in Eqs. (6) and (7) results

$$
\begin{aligned}
& B \int_{0}^{H}\left[a \left\{\frac{\partial}{\partial z}\left(\left(x_{0}-x\right) \frac{\partial u}{\partial z}+\epsilon_{z}{ }^{0}\right\}^{2}\right.\right. \\
& \left.\quad+b\left\{\frac{\partial}{\partial z}\left(\left(x_{0}-x\right) \frac{\partial u}{\partial z}\right)+\epsilon_{z}^{0}\right\}+c\right] d x \\
& \quad+E_{s} \epsilon_{s} A_{s}=0
\end{aligned}
$$

and

$$
\begin{aligned}
& B \int_{0}^{H}\left[a\left\{\frac{\partial}{\partial z}\left(\left(x_{0}-x\right) \frac{\partial u}{\partial z}\right)+\epsilon_{z}{ }^{0}\right\}^{2}\right. \\
& \left.+b\left\{\frac{\partial}{\partial z}\left(\left(x_{0}-x\right) \frac{\partial u}{\partial z}\right)+\epsilon_{2}{ }^{0}\right\}+c\right]\left(d_{1}-x\right) d x \\
& +M+P(L-z)=0
\end{aligned}
$$

Using energy principle as strain energy stored in a system equals to the work done by the external loads acting on the system,

$$
\begin{aligned}
& \int_{V} \int_{0}^{\varepsilon} \sigma_{z}^{\prime} d \epsilon_{z}^{\prime} d v+A_{s} \int_{0}^{L} \int_{0}^{\varepsilon} \sigma_{s}^{\prime} d \epsilon_{s}^{\prime} d z \\
& =(-) \int_{0}^{u} P(u) d u+\int_{0}^{\theta} M(\theta) d \theta
\end{aligned}
$$

where $\sigma_{z}^{\prime}, \sigma_{s}^{\prime}$ are stresses and $\epsilon_{z}^{\prime}, \epsilon_{s}^{\prime}$ are strains corresponding to external load respectively for concrete and steel.

The three Eqs. (9) to (11) contain three unknown $u, x_{0}, \epsilon_{s}$. Boundary conditions are:

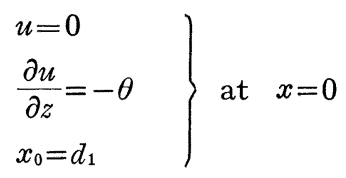

Hence theoretically it is possible to solve the problem of non-bonded pre-stressed member joints. We use numerical technique as the following. For this purpose some more assump- 
tions are made.

(1) For very small change in loads, the loaddeformation curve is assumed to be linear.

(2) The stress-strain relationship for concrete is assumed to be parabolic up to the point of maximum stress and then a straight line up to the ultimate strain as proposed by Hognestad ${ }^{8)}$.

(3) The member is assumed to rotate about its compression edge.

(4) The strain distribution is assumed to be same within an discretized element for the numerical calculation.

\section{(3) Iterative Technique for the Solution of Non- bonded Member Joints}

Note in the beginning that sign convention for concrete stress and strain is changed hereafter to positive for compression. Consider the joint with non-bonded length $L$ of the member as shown in Fig. 1 with load $P$ and moment $M$ at edge of the member. First we assume that moment distribution of non-bonded area is known, though the moment distribution should be iterated for the over all stress-analysis of the system. Equation (2) shows that the displacement $u$ of the non-bonded member is a function of $\theta$, rotation of the joint. For the purpose of analysis, $P$ and $M$ are divided into $N$ number of intervals. Number $N$ is decided depending on the accuracy of the solution desired. Here $N$ is taken to be large enough to make load-deflection and loadrotation curves practically linear between the interval. Thus $\triangle P=P / N$ and $\Delta M=M / N$. Let the steel strain developed due to the load incre-

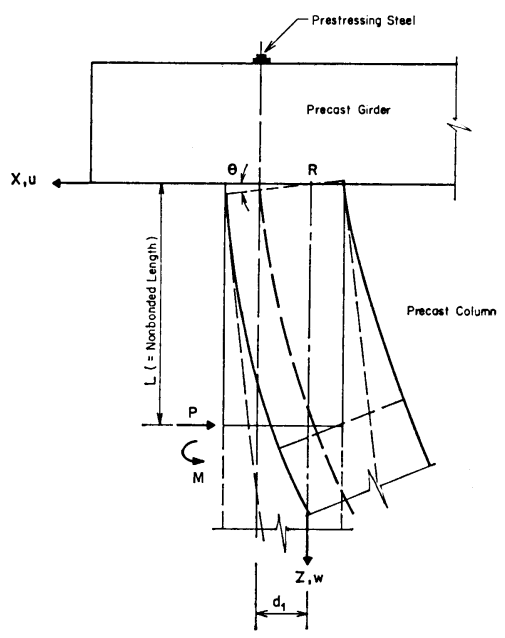

Fig. 1 Joint of Precast Members. ment $\Delta P$ and $\Delta M$ be $\Delta \epsilon_{s}$ and let $\Delta \theta$ and $\Delta u$ be the corresponding rotation of the joint and deflection of the non-bonded member under the load.

At this state of equilibrium, the member will be having some strain energy stored in it. This strain energy stored in the member can be found easily for the assumed steel strain $\epsilon_{s}+\Delta \epsilon_{s}$. As the steel is non-bonded in this area of the member, the compressive force in the concrete being equal to the tensile force in steel, is same throughout the member. Thus location of centre of compression resultant is given by the total moment divided by magnitude of resultant of the compressive force. Knowing compressive force and its location, strain distribution over the section can be found. From the strain distribution, we can find strain energy stored in the concrete. Strain energy stored in steel is also found for the assumed strain in steel. Total energy stored is the sum of the energy stored in concrete and in steel.

From the assumed $\Delta \epsilon_{s}$ i.e. the increased strain in steel, we can find rotation of the joint $\Delta \theta$ and deflection $\Delta u$ under the loads $\Delta P$ and $\Delta M$. Thus at any stage of loading for an assumed $\Delta \epsilon_{s}$ strain increase in steel, it is possible to calculate strain energy developed and the deflection of the non-bonded member under the load. By the principle of energy, strain energy stored in a system is equal to the work done on the system by the external loads.

We iterate the computation assuming differrent values of $\Delta \epsilon_{s}$ until above energy equation is satisfied in the following way.

Let $U$ be the strain energy stored prior to the application of load increments $\Delta P$ and $\Delta M$. If $\Delta U_{T}$ is the net strain energy stored between the load interval $P$ and $P+\Delta P$ and $M$ and $M+$ $\Delta M$ and if the corresponding deflection and rotation at the point of application of loads at the beginning and end of the interval is $u$, $u+\Delta u$ and $\theta_{1}, \theta_{1}+\Delta \theta_{1}$, we have by the principle of energy stated above,

$$
\begin{aligned}
\Delta U_{T}= & (-)\left(\frac{P+P+\Delta P}{2}\right) \Delta u \\
& +\left(\frac{M+M+\Delta M}{2}\right) \Delta \theta_{1}
\end{aligned}
$$

Here load-deflection curve, and load-rotation curve are assumed as linear between the interval $P, P+\Delta P$ and $M, M+\Delta M$ (Fig. 2). $\theta_{1}$ is the rotation at the edge of non-bonded area and is given by $(\theta+\phi)$ where $\phi$ is the rotation of the non-bonded portion of the member due to 


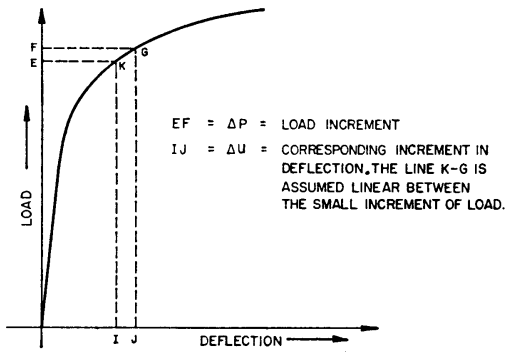

Fig. 2 Typical Load-Deflection Curve.

cantilever action.

$$
\Delta \theta_{1}=\Delta \theta+\Delta \Phi
$$

From Eq. (12)

$$
\frac{\Delta U_{T}}{\Delta u}+\frac{\Delta P}{2}-\left(\frac{M+\Delta M}{2}\right) \frac{\Delta \theta_{1}}{\Delta u}=P
$$

If the assumed value of steel strain increase $\Delta \epsilon_{s}$ satisfies the above equation to the desired degree of accuracy, $u+\Delta u$ and $\theta+\Delta \theta$ are the deflection of the non-bonded member and rotation of the joint for the increase $\Delta P$ and $\Delta M$ in loads. If not some other steel strain $\Delta \epsilon_{s}$ is assumed and whole computation is repeated until the Eq. (13) is satisfied to the desired degree of accuracy. After completing the iteration for the first interval of loading, similar iterative operation, is carried out for second interval treating initial load as $P+\Delta P$ and $M+$ $\Delta M$, initial deformations as $u+\Delta u, \theta+\Delta \theta$ and initial strain energy as $U_{T}+\Delta U_{T}$. The whole process is repeated for all the $N$ intervals to obtain load-deflection and moment rotation curves for the non-bonded member joint.

\section{(4) Strain Distribution Over a Section for a} Given Compressive Force and Its Location

As discussed in previous article we have to obtain strain distribution for the case that location of compressive force is given. Magnitude of compressive resultant is equal to the assumed force in steel due to the non-bonded nature of the wire. Thus the following equations can be written

$$
\begin{aligned}
& M^{\prime}=P(L-z)+M \\
& C=T_{s} \ldots \ldots \ldots \ldots \ldots \ldots \\
& \bar{X}=M^{\prime} / T_{s}
\end{aligned}
$$

Where $M^{\prime}=$ total external moment acting on the section. $M$ and $P$ are moment and shear acting at the edge of non-bonded area. $C$ is the compressive force over the section and $T_{s}$ tensile force in steel. $\bar{X}$ is the distance of centre of com- pressive resultant from the location of prestressing steel.

As already stated the stress-strain curve for concrete is assumed to be as proposed by Hognestad $^{8)}$.

$$
\begin{aligned}
& \frac{\sigma}{\sigma_{\max }}=\frac{2 \epsilon}{\epsilon_{0}}\left(1-\frac{\epsilon}{2 \epsilon_{0}}\right) \\
& \frac{\sigma}{\sigma_{\max }}=1-0.15\left(\frac{\epsilon-\epsilon_{0}}{2 \epsilon_{0}}\right)
\end{aligned}
$$

Here $\sigma_{\max }$ is $0.85 f^{\prime} c$ where $f^{\prime} c$ is the compressive strength of standard cylinders, $\epsilon_{0}$ the strain corresponding to the maximum stress, $\sigma_{\max }$.

According to magnitude and location of centre of compression, the section can experience any one of the five type of stress and strain distributions shown in Figs. 3 to 7.

Case I: $0 \leq \epsilon \leq \epsilon_{0}$ : Fig. 3 .

For this case stress-strain distribution is parabolic at any point in the section.

From Fig. 3, we have

$$
C=\int_{0}^{H} B \sigma d x
$$

where $C$ is the compressive stress resultant, using Eqs. (17) and (19)

$$
\frac{2 B \sigma_{\max }}{\epsilon_{0}} \int_{0}^{H}\left(\epsilon-\frac{\epsilon^{2}}{2 \epsilon_{0}}\right) d x=C
$$

If $\epsilon_{b}$ and $\epsilon_{t}$ are strains in tension face and compression face respectively, strain at any point is

$$
\epsilon=\epsilon_{b}+\left(\frac{\epsilon_{t}-\epsilon_{b}}{H}\right) x
$$

The above equation on substitution in Eq. (20) and after integration results in

$$
\frac{B H \sigma_{\max }}{\epsilon_{0}}\left[\left(\epsilon_{t}+\epsilon_{b}\right)-\frac{1}{3 \epsilon_{0}}\left(\epsilon_{t}{ }^{2}+\epsilon_{b}{ }^{2}+\epsilon_{t} \epsilon_{b}\right)\right]=C
$$

Similarly moment $M^{\prime}$ developed in the section is given by

$$
M^{\prime}=\int_{0}^{H} B \sigma x d x
$$

Using Eq. (17) and then integrating 


$$
\frac{B H^{2} \sigma_{\max }}{3 \epsilon_{0}}\left[\left(2 \epsilon_{t}+\epsilon_{b}\right)-\frac{3 \epsilon_{t}^{2}+\epsilon_{b}^{2}+2 \epsilon_{t} \epsilon_{b}}{4 \epsilon_{0}}\right]=M^{\prime}
$$

Thus the location of $C$ with respect to the gravity point of the prestressing steel is

$$
0.25 H \frac{4 \epsilon_{0}\left(2 \epsilon_{t}+\epsilon_{b}\right)-\left(3 \epsilon_{t}^{2}+\epsilon_{b}^{2}+2 \epsilon_{t} \epsilon_{b}\right)}{3 \epsilon_{0}\left(\epsilon_{t}+\epsilon_{b}\right)-\left\{\left(\epsilon_{t}+\epsilon_{b}\right)^{2}-\epsilon_{t} \epsilon_{b}\right\}}=\bar{X}
$$

From Eqs. (22) and (25), $\epsilon_{t}$ and $\epsilon_{b}$ are obtained for given $C$ and $\bar{X}$. As Eqs. (22) and (25) are nonlinear simultaneous equations, Newton Rapson method was used to solve them.

Case II: $\epsilon_{0} \leq \epsilon \leq \epsilon_{u}$ : Fig. 4

Here $\sigma$ at any point can be expressed by the Eq. (18). If $\sigma_{b}$ and $\sigma_{t}$ are the stress at tension face and compression face then from Fig. 4, we have

$$
B H\left(\frac{\sigma_{b}+\sigma_{t}}{2}\right)=C
$$

Using Eq. (18)

$$
\frac{B H \sigma_{\max }}{\epsilon_{u}-\epsilon_{0}}\left[\left(\epsilon_{u}-0.85 \epsilon_{0}\right)-0.075\left(\epsilon_{t}+\epsilon_{b}\right)\right]=C
$$

Moment $M^{\prime}$ developed in the section is given by

$$
\frac{B H^{2} \sigma_{\max }}{2\left(\epsilon_{u}-\epsilon_{0}\right)}\left[\left(\epsilon_{u}-0.85 \epsilon_{0}\right)-0.05\left(2 \epsilon_{t}+\epsilon_{b}\right)\right]=M^{\prime}
$$

Location of $C$ is,

$$
0.5 H \frac{\left(\epsilon_{u}-0.85 \epsilon_{0}\right)-0.05\left(2 \epsilon_{t}+\epsilon_{b}\right)}{\left(\epsilon_{u}-0.85 \epsilon_{0}\right)-0.075\left(\epsilon_{t}+\epsilon_{b}\right)}=\bar{X}
$$

Thus $\epsilon_{t}$ and $\epsilon_{b}$ are obtained from Eqs. (27) and (29).

Case III: $0 \leq \epsilon_{b} \leq \epsilon_{0}$ and $\epsilon_{0} \leq \epsilon_{t} \leq \epsilon_{u}$ : Fig. 5

Due to linearity of strain distribution

$$
H_{0}=\frac{\epsilon_{0}-\epsilon_{b}}{\epsilon_{t}-\epsilon_{b}} H
$$

where $H_{0}$ is the distance from the tension face to the plane where strain is $\epsilon_{0}$

$$
H^{\prime}{ }_{0}=\frac{\epsilon_{t}-\epsilon_{0}}{\epsilon_{t}-\epsilon_{b}} H
$$

where $H^{\prime}{ }_{0}=H-H_{0}$

Using Eqs. (22) and (27)

$$
\begin{aligned}
& \left(B \sigma_{\max }\right)\left[\frac{H_{0}}{\epsilon_{0}}\left\{\left(\epsilon_{0}+\epsilon_{b}\right)-\frac{\epsilon_{0}{ }^{2}+\epsilon_{b}{ }^{2}+\epsilon_{0} \epsilon_{b}}{3 \epsilon_{0}}\right\}\right. \\
& \left.+\frac{H_{0}{ }^{\prime}}{\epsilon_{u}-\epsilon_{0}}\left\{\left(\epsilon_{u}-0.85 \epsilon_{0}\right)-0.075\left(\epsilon_{t}+\epsilon_{b}\right)\right\}\right]=C
\end{aligned}
$$

Similarly moment $M^{\prime}$ developed is obtained from Eqs. (24), (27) and (29).

$$
\begin{aligned}
& \left(B \sigma_{\mathrm{max}}\right)\left[\frac{H_{0}{ }^{2}}{3 \epsilon_{0}}\left\{\left(2 \epsilon_{0}+\epsilon_{b}\right)-\frac{3 \epsilon_{0}{ }^{2}+\epsilon_{b}{ }^{2}+\epsilon_{0} \epsilon_{b}}{4 \epsilon_{0}}\right\}\right. \\
& +\frac{H_{0}{ }^{\prime}}{\epsilon_{u}-\epsilon_{0}}\left\{\left(\epsilon_{u}-0.85 \epsilon_{0}\right)-0.075\left(\epsilon_{t}+\epsilon_{0}\right)\right\} \\
& \text { - } \left.\left\{H_{0}+0.5 H_{0}^{\prime} \frac{\left(\epsilon_{u}-0.85 \epsilon_{0}\right)-0.05\left(\epsilon_{t}+\epsilon_{0}\right)}{\left(\epsilon_{u}-0.85 \epsilon_{0}\right)-0.075\left(\epsilon_{t}+\epsilon_{0}\right)}\right\}\right] \\
& =M^{\prime}
\end{aligned}
$$

The location of compressive force is,

$$
\begin{aligned}
& {\left[\frac{H_{0}^{2}}{3 \epsilon_{0}}\left\{\left(2 \epsilon_{0}+\epsilon_{b}\right)-\frac{3 \epsilon_{0}{ }^{2}+\epsilon_{b}{ }^{2}+2 \epsilon_{0} \epsilon_{b}}{4 \epsilon_{0}}\right\}\right.} \\
& \quad+\frac{H_{0}^{\prime}}{\epsilon_{u}-\epsilon_{0}}\left\{\left(\epsilon_{u}-0.85 \epsilon_{0}\right)-0.075\left(\epsilon_{t}+\epsilon_{0}\right)\right\} \\
& \left.\cdot\left\{H_{0}+0.5 H_{0}^{\prime} \frac{\epsilon_{u}-0.85 \epsilon_{0}-0.05\left(\epsilon_{t}+\epsilon_{0}\right)}{\epsilon_{u}-0.85 \epsilon_{0}-0.075\left(\epsilon_{t}+\epsilon_{0}\right)}\right\}\right] / \\
& \quad \cdot\left[\frac{H_{0}}{\epsilon_{0}}\left\{\left(\epsilon_{0}+\epsilon_{b}\right)-\frac{\epsilon_{0}^{2}+\epsilon_{b}{ }^{2}+\epsilon_{0} \epsilon_{b}}{3 \epsilon_{0}}\right\}\right. \\
& \left.\quad+\frac{H_{0}^{\prime}}{\epsilon_{u}-\epsilon_{0}}\left\{\epsilon_{u}-0.85 \epsilon_{0}-0.075\left(\epsilon_{t}+\epsilon_{b}\right)\right\}\right]=\bar{X}
\end{aligned}
$$

Eqs. (30) and (32) can again be solved for $\epsilon_{t}$ and $\epsilon_{b}$

Case IV: $0 \geq \epsilon_{b}$ and $0 \leq \epsilon_{t} \leq \epsilon_{0}:$ Fig. 6

$$
H_{1}=\frac{-\epsilon_{b}}{\epsilon_{t}-\epsilon_{b}} H
$$

where $H_{1}$ is the distance of neutral surface from tension face

$$
H_{2}=\frac{\epsilon_{t}}{\epsilon_{t}-\epsilon_{b}} H
$$

where $H_{2}=H-H_{1}$

Neglecting tension in concrete, Eq. (22) on substitution of $H_{2}$ for $H$ and zero, for $\epsilon_{b}$ and after simplification results in

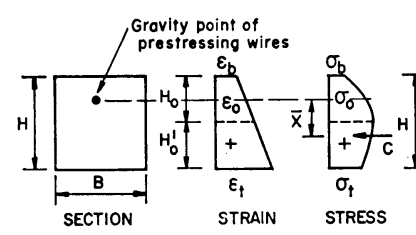

Fig. 5 Strain and Stress Distribution for $0 \leq \epsilon_{b} \leq \epsilon_{0}$ and $\epsilon_{0} \leq \epsilon_{t} \leq \epsilon_{u}$.

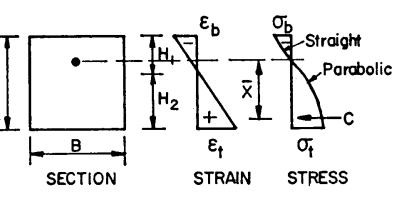

Fig. 6 Strain and Stress Distribution for $0 \geq \epsilon_{b}$ and $0 \leq \epsilon_{t} \leq \epsilon_{0}$.

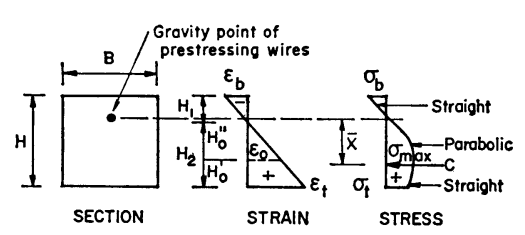

Fig. 7 Strain and Stress Distribution for $0 \geq \epsilon_{b}$ and $\epsilon_{0} \leq \epsilon_{t} \leq \epsilon_{u}$.

Note: In Figs. 3 to 7 , positive sign represents compressive stress and compressive strain. 


$$
\frac{B H}{\epsilon_{0}}\left(\frac{\epsilon_{t}}{\epsilon_{t}-\epsilon_{b}}\right)\left(\sigma_{\max }\right)\left[\epsilon_{t}-\frac{\epsilon_{t}^{2}}{3 \epsilon_{0}}\right]=C
$$

Similarly using Eq. (16)

$$
\frac{-\epsilon_{b}}{\epsilon_{t}-\epsilon_{b}} H+0.25 H\left(\frac{\epsilon_{t}}{\epsilon_{t}-\epsilon_{b}}\right)\left(\frac{8 \epsilon_{0}-3 \epsilon_{t}}{3 \epsilon_{0}-\epsilon_{t}}\right)=\bar{X}
$$

Moment $M^{\prime}$ developed in the section is

$$
\begin{array}{r}
\frac{B H^{2} \sigma_{\max }}{3 \epsilon_{0}^{2}}\left(\epsilon_{t}^{2}\right)\left(\frac{3 \epsilon_{0}-\epsilon_{t}}{\epsilon_{t}-\epsilon_{b}}\right)\left[\frac{-\epsilon_{b}}{\epsilon_{t}-\epsilon_{b}}\right. \\
\left.+0.25 \frac{\epsilon_{t}}{\epsilon_{t}-\epsilon_{b}}\left(\frac{8 \epsilon_{0}-3 \epsilon_{t}}{3 \epsilon_{0}-\epsilon_{t}}\right)\right]=M^{\prime}
\end{array}
$$

Eqs. (33) and (34) is solved to obtain $\epsilon_{t}$ and $\epsilon_{b}$.

Case V: $0 \geq \epsilon_{b}$ and $\epsilon_{0} \leq \epsilon_{t} \leq \epsilon_{u}$ : Fig. 7

$$
H_{2}=\frac{\epsilon_{t} H}{\epsilon_{t}-\epsilon_{b}}
$$

where $\mathrm{H}_{2}$ is the distance of neutral surface from compression face

$$
H^{\prime}{ }_{0}=\frac{\epsilon_{t}-\epsilon_{0}}{\epsilon_{t}} H_{2}
$$

where $H^{\prime}{ }_{0}$ is the distance of the surface where strain is $\epsilon_{0}$ from compression face. Neglecting tension in concrete, $C$ is obtained by substitution of $\epsilon_{b}=0$ and $H_{0}=H^{\prime \prime}{ }_{0}$ in Eq. (30). Here $H^{\prime \prime}{ }_{0}$ is the distance of neutral surface from the surface where strain is $\epsilon_{0}$

$$
\begin{gathered}
\frac{2}{3}\left(B H_{0}{ }^{\prime \prime} \sigma_{\max }\right)+\frac{B H_{0}{ }^{\prime} \sigma_{\max }}{\epsilon_{u}-\epsilon_{0}} \\
\cdot\left[\epsilon_{u}-0.925 \epsilon_{0}-0.07 \epsilon_{t}\right]=C
\end{gathered}
$$

Moment $M^{\prime}$ developed in the section is,

$$
\begin{aligned}
& \frac{2}{3}\left(B H_{0}{ }^{\prime \prime} \sigma_{\max }\right)\left(H_{1}+0.625 H_{0}{ }^{\prime \prime}\right) \\
& +\frac{B H_{0}{ }^{\prime} \sigma_{\max }}{\epsilon_{u}-\epsilon_{0}}\left(\epsilon_{u}-0.925 \epsilon_{0}-0.075 \epsilon_{t}\right) \\
& \text { - }\left(H_{1}+H_{0}{ }^{\prime \prime}+0.5 H_{0}{ }^{\prime} \frac{\epsilon_{u}-0.9 \epsilon_{0}-0.05 \epsilon_{t}}{\epsilon_{u}-0.925 \epsilon_{0}-0.075 \epsilon_{t}}\right) \\
& =M^{\prime}
\end{aligned}
$$

The location of compressive force is

$$
\begin{aligned}
& {\left[\frac{2}{3} H_{0}{ }^{\prime \prime}\left(H_{1}+0.625 H_{0}{ }^{\prime \prime}\right)+\frac{H_{0}{ }^{\prime}}{\epsilon_{u}-\epsilon_{0}}\right.} \\
& \cdot\left(\epsilon_{u}-0.925 \epsilon_{0}-0.075 \epsilon_{t}\right)\left(H_{1}+H_{0}{ }^{\prime \prime}+0.5 H_{0}{ }^{\prime}\right. \\
& \left.\left.\cdot \frac{\epsilon_{u}-0.9 \epsilon_{0}-0.05 \epsilon_{t}}{\epsilon_{u}-0.925 \epsilon_{0}-0.075 \epsilon_{t}}\right)\right] /\left[\frac{2}{3} H_{0}{ }^{\prime \prime}+\frac{H_{0}{ }^{\prime}}{\epsilon_{u}-\epsilon_{0}}\right. \\
& \left.\cdot\left(\epsilon_{u}-0.925 \epsilon_{0}-0.075 \epsilon_{t}\right)\right]=\bar{X} \cdots \cdots \cdots \cdots \cdots(38)
\end{aligned}
$$

Once again Eqs. (36) and (38) can be solved for $\epsilon_{t}$ and $\epsilon_{b}$.

\section{(5) Strain Energy Stored in the Concrete Element}

The pair of equations in each of the five cases yields the strain distribution over the concrete section for given loads. This strain distribution is to be utilized in computing strain energy stored in the section. Strain energy developed over volume $V$ is

$$
U=\int_{V} \int_{0}^{\epsilon}(\sigma d \epsilon) d v
$$

substitution of $\sigma$ from Eqs. (17) and (18) in Eq. (39) using strain distribution obtained in the preceeding article gives strain energy stored in concrete elements for five different cases.

\section{(6) Strain Energy Developed in Prestressing Steel}

Bi-linearized curve shown in Fig. 9 is used for calculation. When the assumed steel strain, $\epsilon_{s}$ is less than yield point $\epsilon_{s y}$, the strain energy developed is

$$
U_{s}=0.5 A_{s} E_{s 1} \epsilon_{s}^{2} L
$$

and when the strain in steel is greater than $\epsilon_{s y}$

$$
\begin{aligned}
U_{s} & =0.5 A_{s} E_{s 1} \epsilon_{s}{ }^{2} L+A_{s} E_{s 1} \epsilon_{s y}\left(\epsilon_{s}-\epsilon_{s y}\right) L \\
& +0.5 A_{s} E_{s 2}\left(\epsilon_{s}-\epsilon_{s y}\right)^{2} L \ldots \ldots \ldots \ldots \ldots \ldots \ldots \ldots \ldots \ldots \ldots \ldots \ldots
\end{aligned}
$$

where $E_{s 1}$ and $E_{s 2}$ are the first and second tangent of bilinearized curve respectively.

\section{(7) Computation of Deflection Under the Load}

For the analysis of the non-bonded member the deflection of the loading point under the load for the assumed steel strain is to be found. Fig. 1 gives the non-bonded member in deflected form after the application of load.

The deflection consists of two parts

(i) deflection considering the column as a cantilever.

(ii) deflection caused by rotation of the joint,

About (i) this deflection is obtained by taking moment of $M / E I$ or $\phi$ diagram about the free end

$$
\delta_{c}=\sum_{i=1}^{N 1}\left(\Phi_{i}\right)\left(x_{i}\right)\left(\Delta x_{i}\right)
$$

where $\phi_{i}$ is the curvature of the element of length $\Delta x_{i}$, located at a distance $x_{i}$ from the free end of the non-bonded member.

For a very small angle $\theta$, the deflection due to rotation as shown in Fig. 2 becomes $L \theta$.

Hence total deflection is,

$$
\delta_{t}=L \theta+\delta_{c}
$$

\section{EXPERIMENTATION}

\section{(1) Manufacturing of Specimen}

In the experimental investigation, only the 
jointing portion of the frame corner is taken out and experimental specimens shows in Fig. 11 were made.

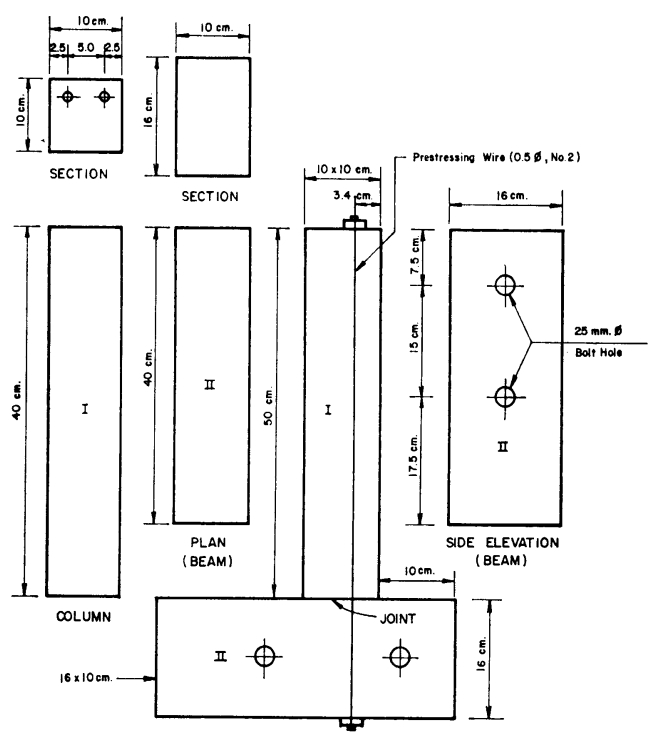

Fig. 8 Assembled Specimen. (Placed Horizontal during Experiment)

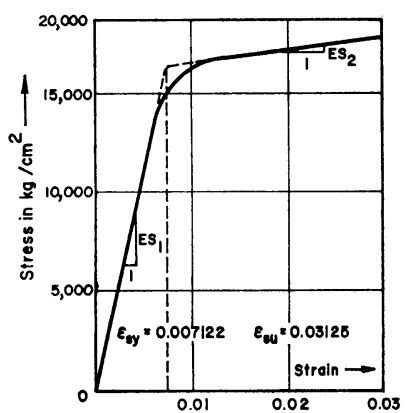

Fig. 9 Experimental Stress-Strain Curve for Prestressing Wire.

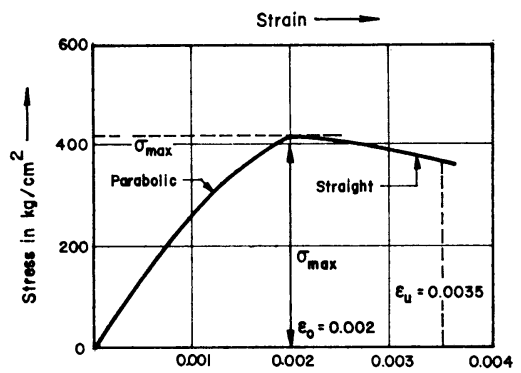

Fig. 10 Experimental Stress-Strain Curve for Concrete.
The assembled specimen shows that it consists of two pre-cast elements. The non-bonded column is $10 \mathrm{~cm} \times 10 \mathrm{~cm} \times 50 \mathrm{~cm}$, with two ducts $(12 \mathrm{~mm} \phi)$ at $3.4 \mathrm{~cm}$ apart from the tension face.

The jointed reinforced concrete precast beam is designed for the anticipated failure load acting at the column top. The sectional dimension of the beam is $10 \mathrm{~cm} \times 16 \mathrm{~cm}$ and its length is $40 \mathrm{~cm}$. Five millimeter diameter high tensile wire is used as a prestressing wire.

For the specimen shown in Fig. 11, electrical strain gauges of $\mathrm{KC} 2$ type are bonded at $10 \mathrm{~cm}$ from each end and at centre of both tension and compression-face of the specimen. The arrangement of strain gauges is also shown in Fig. 11 . Similarly three PL-5 type strain gauges are attached to each of the two $0.5 \mathrm{~cm}$ diameter high tensile wires at $20 \mathrm{~cm}, 35 \mathrm{~cm}$, and $50 \mathrm{~cm}$ apart from one end of the wire.

The specimen is then positioned as shown in Fig. 12. Each of the two steel wire with strain gauges and soldered electrical wires are inserted into the two ducts of the non-bonded member and in the beam. Four steel end plates $(5 \mathrm{~cm} \times$ $5 \mathrm{~cm} \times 0.6 \mathrm{~cm})$ are then inserted along with 4 Freyssinet cones.

Cylinders of the mix tested at 14 days gave

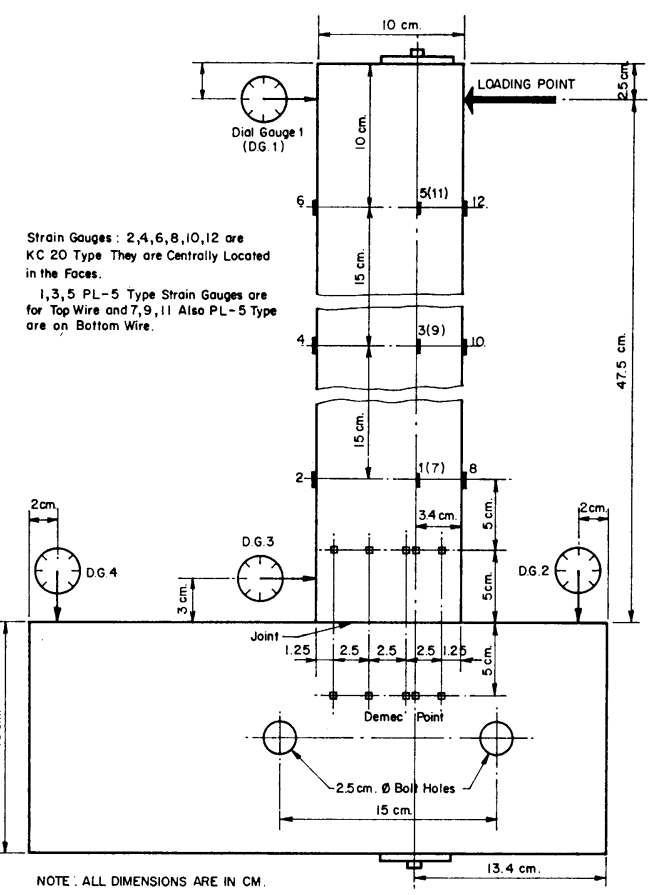

Fig. 11 Specimen with Attached Dial Gauges and Demec Gauges. 


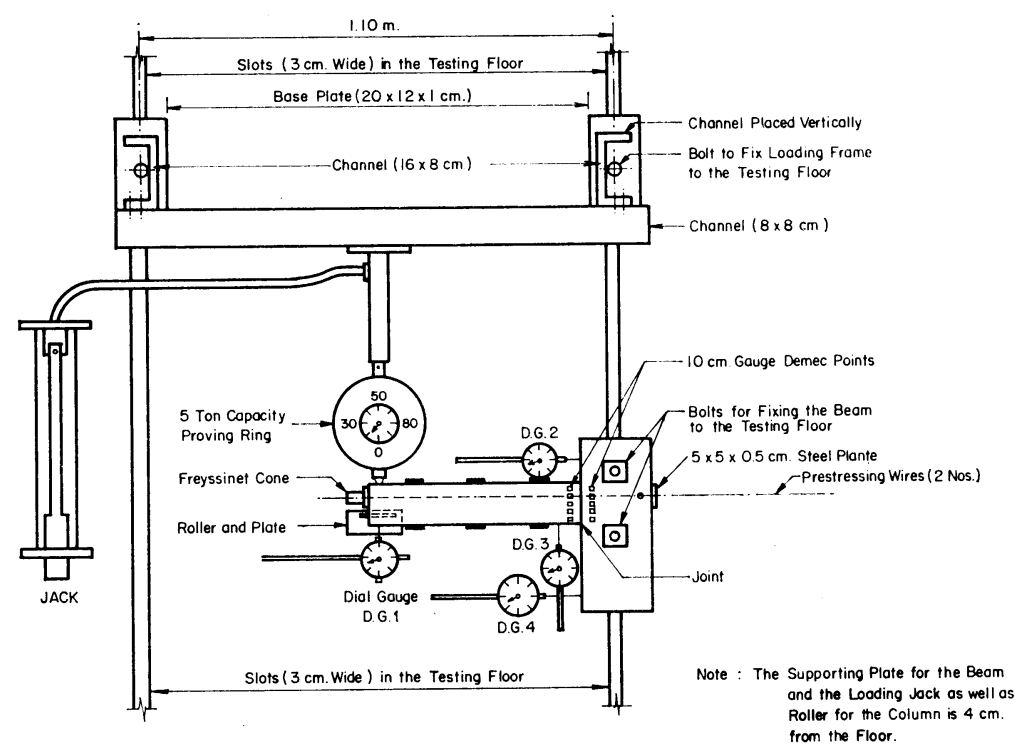

Fig. 12 Plan of Set Up of The Test.

$439 \mathrm{~kg} / \mathrm{cm}^{2}$ as mean cylinder strength. The typical stress-strain curve obtained is shown in Fig. 10.

\section{(2) Testing of the Specimen}

At first initial reading of bonded demec gauges and attached dial gauges are taken.

A five ton capacity jack is positioned to apply the concentrated load at locaton $2.5 \mathrm{~cm}$ apart from the free end of the non-bonded member. Load is applied slowly at the interval of $50 \mathrm{~kg}$ until it failed.

Readings for strain gauges, dial gauges and demec gauges are noted down for each interval of load. Readings are taken till the specimen fails.

\section{RESULTS AND DISCUSSION}

In Figs. 13 to 16 the experimental results are shown, namely moment vs. rotation, load vs. deflection, load vs. separation of joint, load vs. steel strain.

The theoretically computed curves are obtained by utilizing the experimentally tested properties of concrete and steel. For the purpose of theoretical analysis, the non-bonded portion $(10 \mathrm{~cm} \times$ $10 \mathrm{~cm} \times 50 \mathrm{~cm}$ ) was divided into 10 identical elements $(10 \mathrm{~cm} \times 10 \mathrm{~cm} \times 5 \mathrm{~cm}$ high $)$. Properties of the wires used are taken from bilinearized experimental curve shown in Fig. 9. The slopes of steel stress-strain curves for elastic and inelastic portions from the same curve, are $2 \times 10^{6}$

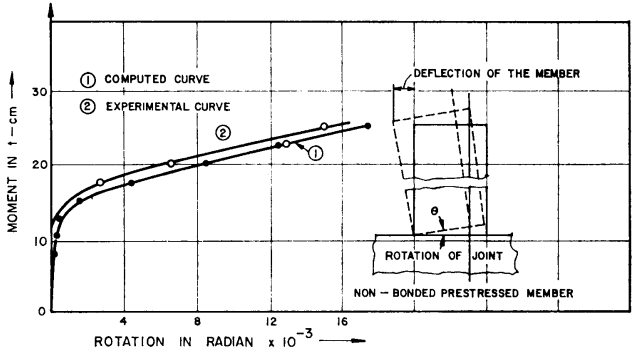

Fig. 13

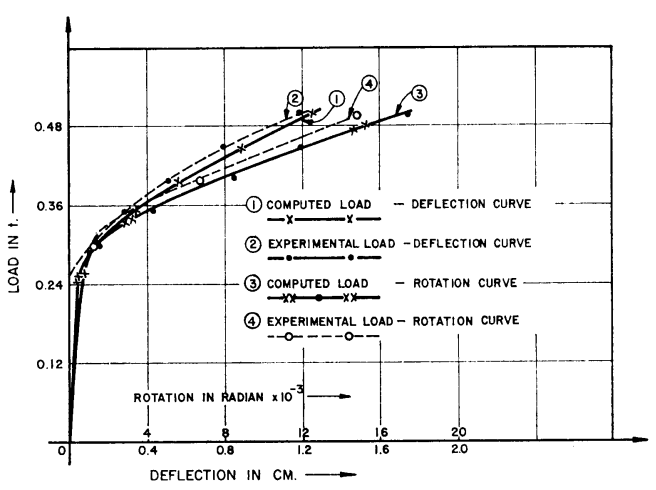

Fig. 14

$\mathrm{kg} / \mathrm{cm}^{2}$ and $58 \mathrm{~kg} / \mathrm{cm}^{2}$ respectively. The apparent yield strain and ultimate strain taken from same curve, are 0.00722 and 0.03125 respectively. Similarly properties of concrete 


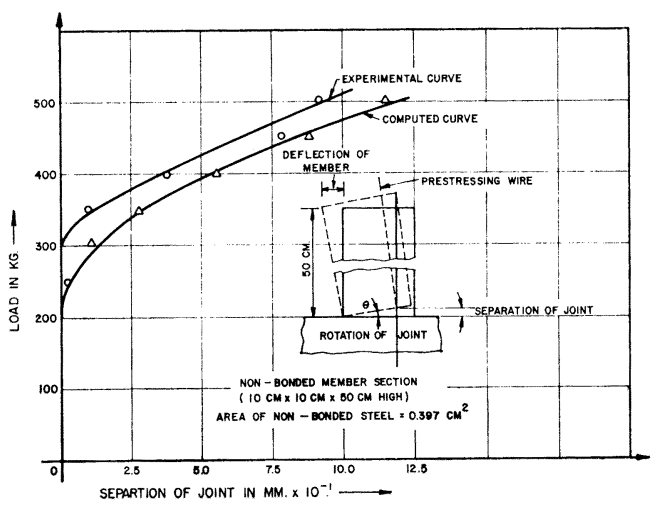

Fig. $\llbracket 15$

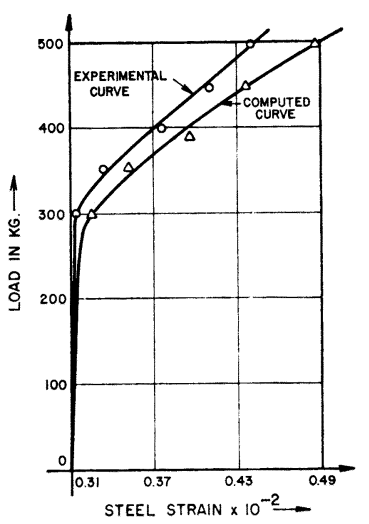

Fig. 16

used for the computation are taken from the idealized experimental stress-strain curve of Fig. 10. Strain at the maximum stress is $\epsilon_{0}=0.002$ and ultimate strain is $\epsilon_{u}=0.0035$. The computation is made at $50 \mathrm{~kg}$ interval of loading and initial prestrain applied is 0.0031 . As defined by Hognestad, stress corresponding to $\epsilon_{0}$ is $0.85 \times$ (cylinder strength of concrete used). It is equal to $360 \mathrm{~kg} /$ $\mathrm{cm}^{2}$.

Computed curves as well as experimental curves of moment vs. joint rotation and load vs. deflection under the load are shown in Fig. 13 and Fig. 14 respectively. The curves of moment vs. joint rotation shows that there is reasonable agreement between the computed curve and the experimental curve. Similar is the case with load vs. deflection and load vs. rotation curves. From these curves, it is clearly seen that there exists a loading point after which rotation and deflection suddenly increase very rapidly. It is found from computation as well as experimentation that this point corresponds to the load which give rise tension stress in the section. Observing load vs. steel strain curve of load-separation curve, shown in Fig. 15 and Fig. 16 respectively, it is again clear that before the balanced load*, there is no separation of joint in case of experimental curve and very little separation in case of computed curve. Also there is very little increase in steel strain as shown by computed as well as experimental curves of Fig. 16, computed curve giving slightly greater values. After the balanced load*, separation becomes prominent and strain in the steel increases rapidly. This consequently results in the increased rotation of the joint and deflection of the non-bonded member. It is thus observed that experimental curves and computed curves for moment vs. rotation, load vs. deflection, load vs. separation of joint and load vs. steel strain agree fairly well.

The computation assumes a constant value for the location of centre of rotation at the joint. The centre of rotation assumed for computation is along the compression edge of the non-bonded member at the joint. As the load applied get slightly more than balanced load* the centre of compression shifts towards the compression edge as much as the section can accommodate. As prior to this load rotation obtained is negligibly small, the error involved can also be neglected and after this load the assumption is sufficiently accurate. In regard to the assumption of neglecting change in length of concrete in comparison with steel, the agreement of the experimental and computed curves shows that the error involved due to such assumption can be safely neglected for practical purposes, as the change in concrete strain is very less in comparison with change in steel strain for the same external load. Despite the slight discrepancies due to above assumptions the solution has been able to predict the behaviour of non-bonded prestressed member joint in a satisfactory manner as illustrated by the curves shows in Figs. 13 to 16 .

To demonstrate the difference of rigidity characteristic of non-bonded joints and bonded joints, deflection of the bonded specimen, dimension and material properties of which are taken as same as non-bonded specimens was calculated using linear stress and strain variation over the section. The plots of computed values of load vs. deflection for non-bonded and bonded type is shown in Fig. 17. Observing the curves, both of them increase rapidly giving greater deflection

* A loading point after which rotation and deflection suddenly increase very rapidly. 


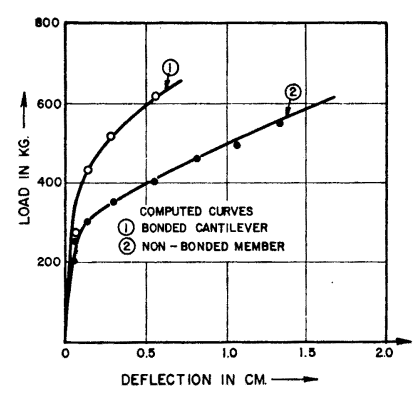

Fig. 17

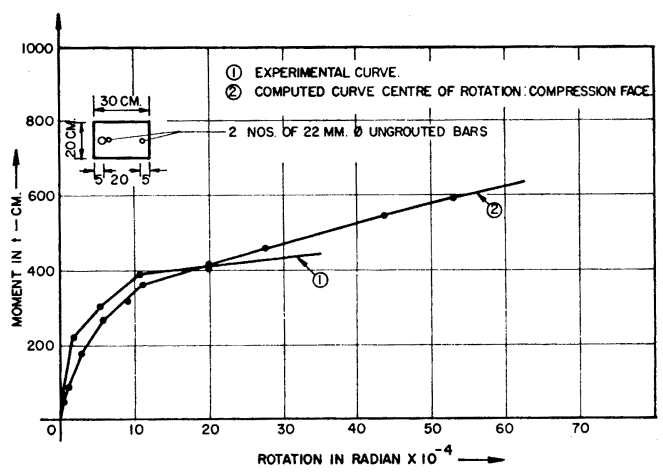

Fig. 18

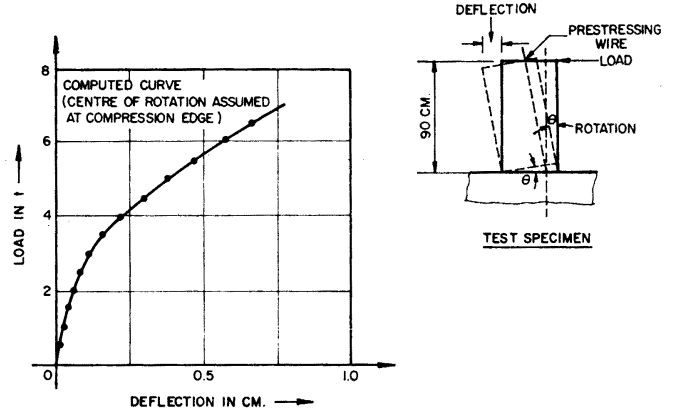

Fig. 19

when applied pre-strain can no longer counteract the external applied load. Prior to this load, deflection obtained in both cases are small, nonbonded one exhibiting slightly greater deflection than the bonded one. After this loading, the increase in deflection of non-bonded member is much greater than those of bonded member. The plot further shows that the rigidity of nonbonded and that of bonded type are almost same up to the balance load*. Beyond this load, the curves show that non-bonded case has very low rigidity compared to bonded type. This is at- tributed to the different distributions of steel strains in the two types considered. The increase in steel strain in non-bonded member is large and is same throughout the length whereas the increase in steel strain in bonded member is equal to the tensile strain in concrete at the level of steel. The greater increase in steel strain causes larger rotation of the member at the joint and thus increasing the deflection.

It is also clear that at ultimate load the curve 2 for non-bonded joints shows nearly 2.5 times larger deflection of the curve 1 for bonded joints.

The same analysis was carried out for larger sized specimen shown in Fig. 18; of which experiments was conducted by T. Tanabe ${ }^{9)}$. For the purpose of analysis the non-bonded member of $90 \mathrm{~cm}$ height was divided into 18 elements of $5 \mathrm{~cm}$ height. For steel, bilinearized stress-strain curve is used with slopes of $2 \times 10^{6} \mathrm{~kg} / \mathrm{cm}^{2}$ and $58.9 \mathrm{~kg} / \mathrm{cm}^{2}$ for elastic and inelastic portion respectively. The apparent yiel strain and ultimate strain is taken as 0.00722 and 0.03125 respectively. For concrete, parabolic stress-strain curve as proposed by Hognestad is taken. The strain at maximum stress of concrete is taken as 0.002 and its ultimate strain is taken as 0.0035. Stress at maximum strain is taken as $300 \mathrm{~kg} / \mathrm{cm}^{2}$. Moment-rotation and load-deflction characteristics are computed at every $1 / 2 \mathrm{t}$ interval. The centre of rotation is also taken at compression edge of the member at the joint.

The computed curve as well as experimental curve of moment-rotation and load-rotation ${ }^{9}$ are shown in Fig. 18. From the curves it is observed that basic nature of the computed curve resemble to that of experimental one. The assumption that centre of rotation lies at the compression edges produces reliable results is also clear from the curves. In the experiment the rotation was determined by a series of demec readings across the joint and then taking the mean of the rotations obtained between the successive demec gauges. The very little deviation between the computed curve and experimental curve justifies the assumption the centre of rotation at the compression face near the joint for the non-bonded member for all practical purposes.

\section{CONCLUSION}

From the foregoing discussion of the results of the investigation it can be concluded that,

(a) The method of analysis developed, satis- 
factorily predicts

(i) moment-rotation characteristics of the non-bonded prestressed precast joints,

(ii) load-deflection characteristics of the non-bonded member.

(b) The rotation of prestressed precast joints is negligibly small until the applied load exceed the balanced load* for both nonbonded and bonded members. After the balanced load* as the centre of compression is already shifted near the compression edge at the joint, any increase of load results comparatively greater increase in steel strain than in fully prestressed condition. However, due to this increased steel strain throughout non-bonded length of the member, and thus the increased length of steel wire, the rotation of the joint and the deflection of the non-bonded member increase more rapidly than a bonded member. The rapid decrease of the joint rigidity of non-bonded joints may be accurately estimated by the proposed method of analysis. This will help to analyze the total system of frame considering the effect of joint rotation.

(c) This type of joint clears by itself the effect of overload (provided neithter concrete nor steel has failed) as soon as the overload is removed. The separation of the joint and the deflection of the non-bonded member will vanish if the load is reduced. The appearance of the joint after the reduction of the load shows no signs of applied overload. This is considered to be an added advantage of this type of structures.

\section{NOTATION}

$A_{s}=$ area of prestressing steel

$B=$ width of the section

$C=$ compressive force in concrete

$E_{\boldsymbol{s} 1}=$ modulus of elasticity of pre-stressing wire

$E_{s 2}=$ slope of the stress-strain curve of pre-stressing wire for inelastic portion

$f^{\prime}{ }_{c}=$ compressive strength of concrete cylinder

$H=$ depth of the section

$H_{0}=$ the distance from tension face to the surface where strain is $\epsilon_{0}$

$H^{\prime}{ }_{0}=$ the distance of the compression face from the surface where strain is $\epsilon_{0}$

$H^{\prime \prime}{ }_{0}=$ the distance of neutral surface from the surface where strain is $\epsilon_{0}$
$H_{1}=$ the distance of neutral surface from tension face

$H_{2}=$ the distance of neutral surface from the compression face

$L=$ length of the non-bonded member

$M=$ external moment acting at the edge of non-bonded portion

$M^{\prime}=$ bending moment at any section

$N=$ number of interval into which loads are divided

$N_{1}=$ number of divided elements in nonbonded member

$P=$ load acting at the edge of the nonbonded portion

$U=$ strain energy in concrete element

$U_{s}=$ strain energy in pre-stressing steel

$U_{T}=$ total strain energy

$u=$ displacement along $X$-axis

$v=$ displacement along $Y$-axis

$w=$ displacement along $Z$-axis

$\Delta M=$ increment of moment acting at the edge of non-bonded member

$\Delta P=$ increment of load acting at the edge of non-bonded portion

$\Delta U_{T}=$ increase of total strain energy due to load increment

$\Delta u=$ increment of displacement along $X$ axis

$\Delta x=$ height of the element

$\boldsymbol{\delta}_{\boldsymbol{c}}=$ deflection under the applied load considering the non-bonded member as a cantilever

$\delta_{t}=$ total deflection under the load

$\epsilon=$ strain at any section

$\epsilon_{b}=$ strain on the tension face of concrete

$\epsilon_{c}=$ strain of concrete

$\epsilon_{0}=$ strain corresponding to maximum compressive stress in concrete

$\epsilon_{s}=$ strain of steel

$\epsilon_{t}=$ strain of concrete on the compression face

$\epsilon_{u}=$ ultimate compressive strain of concrete

$\epsilon_{z}=$ strain along $Z$-axis

$f^{\prime}{ }_{c}=$ compressive strength of concrete cylinder

$\sigma=$ stress in concrete

$\sigma_{b}=$ stress in the tension face of concrete

$\sigma_{t}=$ stress in the compression face of concrete

$\sigma_{\max }=$ maximum stress in concrete as per Hognestad's curve 


\section{REFERENCES}

1) Rostasy, F. S.: "Connections in Precast Concrete Structures-Continuity in Double -T Floor Construction", Journal of the Prestressed Concrete Institute, Vol. 7, No. 4, pp. 18 48; PCA Development Department Bullein D55 1962.

2) Kriz, L. B. and C. H. Raths: "Connections in Precast Concrete Structures-Bearing Strength of Column Heads", Journal of the Prestressed Concrete Institute, Vol. 8, No. 6 , pp. $45 \sim 75$; PCA Development Department Bulletin D73, 1963.

3) Kriz, L. B. and C. H. Raths: "Connections in Precast Concrete Structures-Strength of Corbels", Journal of the Prestressed Concrete Institute, Vol. 10, No. 1, pp. 16 61; PCA Development Bulletin D85, 1965.

4) Gaston, J. R. and L. B. Kriz: "Connections in Precast Concrete StructuresTest of Scarf Joints", Journal of the Prestressed Concrete Institute, Vol. 9, No. 3, pp. 37 59; PCA Development Department Bulletin D79, 1964.

5) Laeraugh, R. W. and D. D. Magura: "Connections in Precast Concrete StructuresColumn Base Plates", Journal of the Prestressed Concrete Institute, Vol. 11, No. 6, pp. 18 35; PCA Development Department Bulletin D110, 1966.

6) Burns, N. H.: "Development of Continuity Between Prestressed Concrete Beams", Journal of the Prestressed Concrete Institute, Vol. 11, No. 3, pp. 23 36, 1966.

7) Schechter, E. and M. Suarez: "Post-tentioned Anchorages for Engineered Structures", ACI Publication SP-22, Mechanical Fasteners for Concrete, pp. 139 160, 1969.

8) Hognestad, E.: "A Study of Combined Bending and Axial Load in Reinforced Concrete Members", Bulletin No. 399, Urbana, Illinois, University of Illinois Engineering Experiment Station, pp. 128, Nov. 1951.

9) Tanabe, T.: "Fundamental Study on the Use of Precast Concrete Members in Concrete Composite Structure", Proceedings of the Japan Society of Civil Engineers, No. 206, pp. 111, Oct. 1972.

10) AIT, R. C. C.: "Solution to a Set of Nonlinear Equations Subroutine Program", pp. $3 \sim 5$.

11) ACI Committee 211: "Recommended Practice for Selecting Proportions for Normal Weight Concrete", ACI Journal, Title No. 66-49, pp. 612 618, August 1969.

(Received December 8, 1977) 